\title{
Mortality of septic shock patients is associated with impaired mitochondrial oxidative coupling efficiency in lymphocytes: a prospective cohort study
}

\author{
Wagner Luis Nedel ${ }^{1,2}$, Afonso Kopczynski ${ }^{1}$, Marcelo Salimen Rodolphi ${ }^{1}$, Nathan Ryzewski Strogulski ${ }^{1}$, \\ Marco De Bastiani ${ }^{3}$, Tiago Hermes Maeso Montes², Jose Abruzzi Jr², Antonio Galina ${ }^{4}$, Tamas L. Horvath ${ }^{5}$ and \\ Luis Valmor Portela ${ }^{1 *}$ (1)
}

\author{
${ }^{*}$ Correspondence: \\ roskaportela@gmail.com \\ ' Laboratory of Neurotrauma \\ and Biomarkers, \\ Departamento de \\ Bioquímica, Programa \\ de Pós-Graduação \\ em Bioquímica, ICBS, \\ Universidade Federal do Rio \\ Grande do Sul, Rua Ramiro \\ Barcelos, 2600, anexo, Porto \\ Alegre, RS, Brazil \\ Full list of author information \\ is available at the end of the \\ article
}

\begin{abstract}
Background: Septic shock is a life-threatening condition that challenges immune cells to reprogram their mitochondrial metabolism towards to increase ATP synthesis for building an appropriate immunity. This could print metabolic signatures in mitochondria whose association with disease progression and clinical outcomes remain elusive.
\end{abstract}

Method: This is a single-center prospective cohort study performed in the ICU of one tertiary referral hospital in Brazil. Between November 2017 and July 2018, 90 consecutive patients, aged 18 years or older, admitted to the ICU with septic shock were enrolled. Seventy-five patients had Simplified Acute Physiology Score (SAPS 3) assessed at admission, and Sequential Organ Failure Assessment (SOFA) assessed on the first (D1) and third (D3) days after admission. Mitochondrial respiration linked to complexes $I, I I, V$, and biochemical coupling efficiency (BCE) were assessed at D1 and D3 and $\triangle$ (D3-D1) in isolated lymphocytes. Clinical and mitochondrial endpoints were used to dichotomize the survival and death outcomes. Our primary outcome was 6-month mortality, and secondary outcomes were ICU and hospital ward mortality.

Results: The mean SAPS 3 and SOFA scores at septic shock diagnosis were 75.8 $( \pm 12.9)$ and $8( \pm 3)$ points, respectively. The cumulative ICU, hospital ward, and 6-month mortality were $32(45 \%), 43$ (57\%), and 50 (66\%), respectively. At the ICU, nonsurviving patients presented elevated arterial lactate ( $2.8 \mathrm{mmol} / \mathrm{L}, \mathrm{QQR}, 2-4)$, C-reactive protein (220 mg/L, IQR, 119-284), and capillary refill time (5.5 s, IQR, 3-8). Respiratory rates linked to $C I I$ at $D 1$ and $D 3$, and $\triangle C$ II were decreased in non-surviving patients. Also, the BCE at D1 and D3 and the $\triangle B C E$ discriminated patients who would evolve to death in the ICU, hospital ward, and 6 months after admission. After adjusting for possible confounders, the $\triangle B C E$ value but not SOFA scores was independently associated with 6-month mortality (RR 0.38, Cl 95\% 0.18-0.78; $P=0.009$ ). At a cut-off of -0.002 , $\triangle B C E$ displayed $100 \%$ sensitivity and $73 \%$ specificity for predicting 6-month mortality author(s) and the source, provide a link to the Creative Commons licence, and indicate if changes were made. The images or other third party material in this article are included in the article's Creative Commons licence, unless indicated otherwise in a credit line to the material. If material is not included in the article's Creative Commons licence and your intended use is not permitted by statutory regulation or exceeds the permitted use, you will need to obtain permission directly from the copyright holder. To view a copy of this licence, visit http:// creativecommons.org/licenses/by/4.0/. 
Conclusions: The $\triangle B C E$ signature in lymphocytes provided an earlier recognition of septic shock patients in the ICU at risk of long-term deterioration of health status.

Keywords: Septic shock, Lymphocytes, Mitochondrial signatures, Mortality, Prognostic biomarker

\section{Introduction}

Sepsis is a disorder that develops as organ disfunction, and remains one of the leading causes of death globally. Although it may benefit from early diagnostic and prompt treatments to minimize mortality, the availability of diagnostic tools to better predict outcomes and support early clinical decisions are still limited. Septic shock, the most severe end of the spectrum of sepsis, represents a life-threatening condition, which requires vasopressor therapy to treat profound circulatory and metabolic abnormalities [1, 2]. Indeed, during septic shock the oxidative metabolism in several tissues may be limited by the oxygen saturation, delivery, and utilization by mitochondria. Therefore, an early antibiotic therapy, fluid resuscitation and vasopressor drugs alone may not overcome the respiratory deficits at the cellular level. This highlights that inherent or acquired mitochondrial defects associated with septic shock may hinder oxygen consumption coupled with ATP synthesis (OXPHOS), independent of standard therapeutic interventions, thereby contributing to mechanisms underlying organ failure and, eventually, to patient's death [3-5].

Current advances in the understanding of the physiopathology of sepsis have incorporated the concept of an existing crosstalk between immune cells metabolism and immunity [5-9]. The inflammatory course of septic shock challenges the immune cells to enhance their production of antibodies and signaling molecules over time, which relies on an increased energy consumption supported by the mitochondrial metabolic machinery $[7,10]$. Based on this, mitochondria of lymphocytes are constantly reprogramming their metabolism, with the increased activity of oxidative complexes reflecting their higher requirements for ATP synthesis by working at high respiratory rates or the downregulation of oxidative complexes reflecting decreased ATP demands or mitochondrial metabolic exhaustion [9]. These unique features suggest that mitochondria might print metabolic signatures in immune cells of septic shock patients whose clinical significance as a biomarker remains elusive $[4,5,11-14]$. Remarkably, the rates of oxygen consumption associated with individual mitochondrial complexes can be profiled in lymphocytes by respirometry protocols allowing to derive a composite of metabolic signatures that may serve as candidate biomarkers of clinical outcomes. Particularly, the BCE is a parameter which scores how efficiently the mitochondrial machinery are synchronized towards ATP-synthesis $[15,16]$.

Early studies have analyzed OXPHOS metabolism in sepsis, with controversial results mainly regarding the capability of metabolic endpoints to predict organ failure and mortality $[4,5,11-14,17]$. In general, they focused on sepsis and did not include a large number of patients with septic shock, which reduced the power of clinical associations between organ failure, mortality, the effectiveness of therapies and specific mitochondrial respiratory states. Also, few studies conducted a strategy of two sequential assessments in the ICU setting and following its impact on the short-, mid- and long-term prognosis. 
Therefore, there are still gaps to fill regarding mitochondrial metabolic signatures in immune cells as feasible biomarkers for septic shock patients admitted to the ICU. Accordingly, we aimed to investigate mitochondrial bioenergetic signatures in lymphocytes associated with mortality for septic shock.

\section{Material and methods}

\section{Participants and study design}

This is a prospective cohort study designed to evaluate the mitochondrial metabolism of circulating lymphocytes in septic shock patients admitted to four different ICUs from one tertiary university public hospital in Brazil. This study was approved by the local ethics committee (Plataforma Brazil number 66240017.0.0000.5530). We prospectively enrolled 90 adult patients ( $>18$ years old, $45 \%$ were women) admitted to the ICU due to septic shock between November 2017 and July 2018 as indicated in Fig. 1. Patients were excluded if they presented with a known mitochondrial disease, pregnancy, refusal of the patient or the next of kin to sign the informed consent, patients with imminent death, and patients with withholding or withdrawing treatments. Septic shock was defined as the presence of persistent hypotension with the requirement of vasopressor therapy to maintain a mean arterial pressure of $65 \mathrm{mmHg}$ or greater according to Sepsis 2001 definition [18]. The following demographic and clinical characteristics were prospectively recorded: gender, age, primary site of infection, community-acquired or hospital-acquired infection, comorbidities, noradrenaline maximum dose, lactate level,

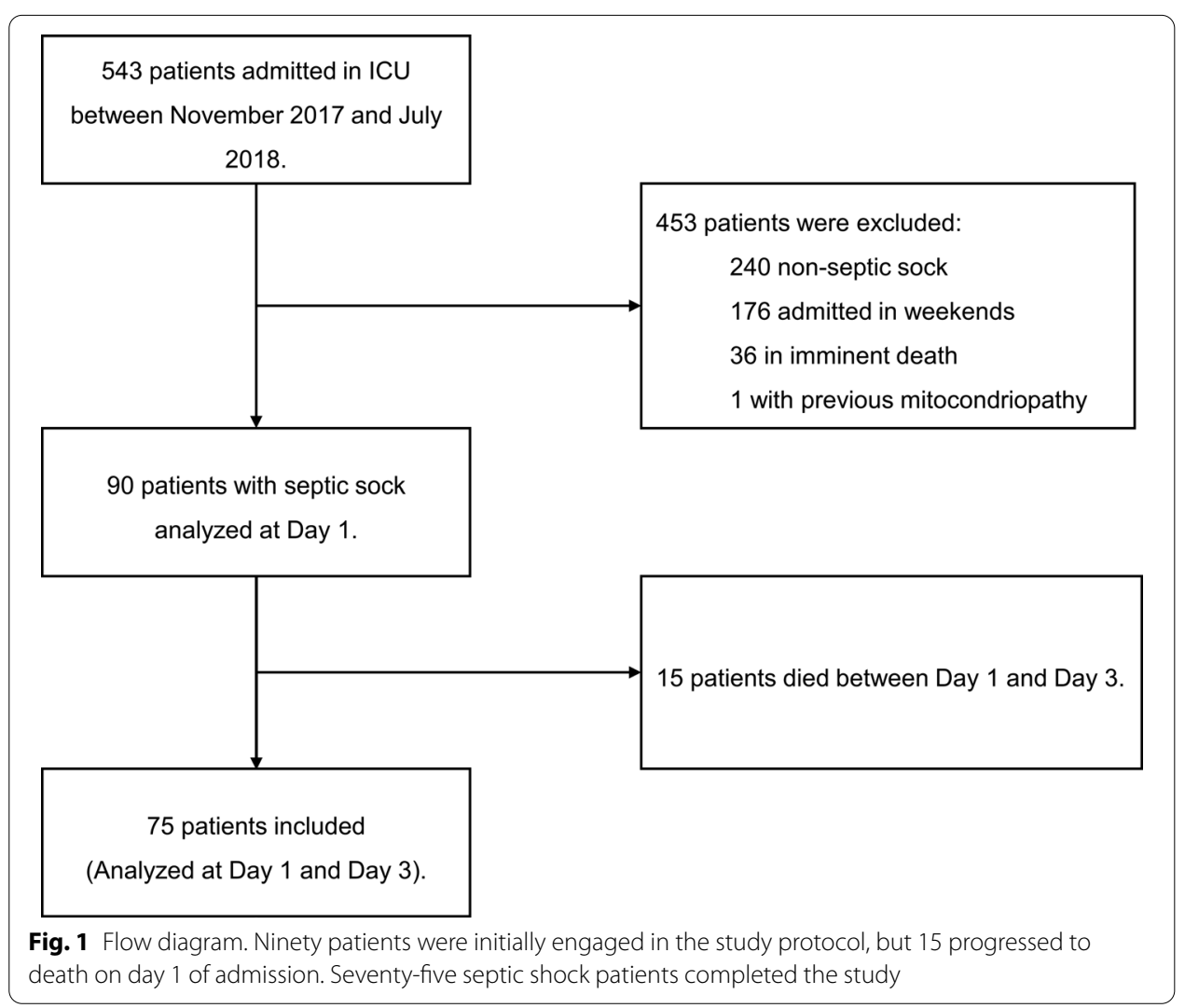


urine output, $\mathrm{PaO}_{2}: \mathrm{FiO}_{2}$ ratio, serum creatinine, total bilirubin, platelets, international normalized rate (INR), Glasgow Coma Scale, capillary refill time, central venous saturation $\left(\mathrm{SvO}_{2}\right)$, length of ICU and length of hospital stay. Also, Simplified Acute Physiology Score (SAPS 3) and the Sequential Organ Failure Assessment (SOFA) score were assessed $[19,20]$. The SOFA score was used as a tool for defining both, the clinical condition of the individual patient and the response to therapies [21], as well as delta SOFA as an indicator of resolved or unresolved clinical condition [22]. This score is a gold standard for predicting hospital mortality, providing detailed information regarding the number and severity of organic failure in septic patients [23]. The management of septic shock in these patients was carried out as recommended by Surviving Sepsis Campaign [24] guidelines, especially with regard to early antibiotic therapy, culture collection, fluid replacement and preferential use of norepinephrine as first-line vasopressor drug.

Clinical and laboratory endpoints, including mitochondrial respirometry in permeabilized lymphocytes (digitonin $0.005 \% \mathrm{w} / \mathrm{v}$ ), were evaluated at two timepoints: the first day of ICU admission (D1) and three days after admission (D3). The pairwise variations between D1 and D3 $(\Delta)$ were used to estimate the improvement or the worsening of the clinical, laboratory and mitochondrial endpoints. SOFA and mitochondrial endpoints were used to dichotomize the survival and death outcomes. The primary outcome was 6-month mortality for septic shock, and the secondary outcomes were ICU and hospital mortality.

\section{Isolation of lymphocytes and mitochondrial respirometry}

Six milliliters of blood were sampled in EDTA tubes at D1 and D3. Lymphocytes were isolated as described by Pecina et al. [25]. We assayed mitochondrial respiration in permeabilized lymphocytes isolated at D1 and D3. The cells counting showed $98 \%$ of the lymphocytes in the fraction.

The high-resolution respirometry measurements were performed within $3 \mathrm{~h}$ of blood sampling using oxygraphy (Oxygraph-2 k; Oroboros Instruments, Innsbruck, Austria) at $37{ }^{\circ} \mathrm{C}$. Oxygen concentration (micromolar) and oxygen flux (expressed in $\mathrm{pmol} \mathrm{O}_{2}$. $\mathrm{s}^{-1}$.mg of protein) were recorded with DatLab software 6.0. The basal oxygen consumption (oxygen flow per volume or per mass) was established without metabolic substrates. Subsequently, stepwise additions of pyruvate, malate and glutamate $(10,10$ and $20 \mathrm{mM}$, respectively), followed by $2.5 \mathrm{mM} \mathrm{ADP}, 10 \mathrm{mM}$ succinate, a second $2.5 \mathrm{mM} \mathrm{ADP}$, and finally sodium azide plus antimycin A were performed. This protocol assesses the respiration linked to Complex I (CI), Complex II (CII) and maximal oxygen flow rate consumption coupled to ATP production (P), and nonmitochondrial oxygen consumption [26].

The $\mathrm{BCE}$ (also known as $\approx \mathrm{P}$ control factor) was measured to estimate the mitochondrial oxygen flow coupled to ATP production. The BCE is calculated by the $\mathrm{P}-\mathrm{L} / \mathrm{P}$ fluxes; $(\mathrm{J} \approx \mathrm{P}=(\mathrm{P}-\mathrm{L}) / \mathrm{P})$, as described previously $[15,16]$. A representative image displaying the sequential addition of the substrates to the permeabilized lymphocytes, and the derived respiratory parameters obtained from a control subject, are shown in Additional file 1: Figure S1. The researchers involved in the mitochondrial analysis were blinded to the clinical outcomes, and the researchers involved in the clinical data collection were blinded to the mitochondrial outcomes. All chemicals used for high-resolution 
respirometry analysis were analytical grade, purchased from Sigma-Aldrich (SigmaAldrich, St. Louis, MO, USA).

\section{Statistical analysis}

Descriptive statistics included frequencies and percentages for the categorical variables and means, and standard deviation, confidence intervals, medians, and interquartile ranges for continuous variables. Student's $t$-test or the Mann-Whitney $U$ test were used to compare continuous variables according to normality, assessed by Shapiro-Wilk test. Chi-square test or Fisher's exact test were used to analyze categorical variables. Association between two continuous variables was measured with Spearman correlation coefficient. To access the impact of $\mathrm{BCE}$ improvement at $\mathrm{D} 3(\triangle \mathrm{BCE}>0)$ on the outcomes, Poisson regression was performed with 6-month mortality as the dependent variable, and hematological neoplasia, chronic kidney disease, lactate at septic shock diagnosis, SOFA improvement at D3 $(\triangle \mathrm{SOFA}<0)$ and the SAPS 3 score at ICU admission as independent variables in the model. These variables were selected for the model because they presented a $\mathrm{p}$ value of less than 0.20 in the univariate analysis. In this statistical model $\triangle$ basal, $\triangle \mathrm{CI}$ and $\triangle \mathrm{CII}$, were not included since they are partial components of the BCE. $\triangle$ SOFA score was dichotomized between improvement $(\triangle \mathrm{SOFA}<0)$ and worsening $(\triangle \mathrm{SOFA}>0)$ on $\mathrm{D} 3$, rather than using its value in $\mathrm{D} 1$ and $\mathrm{D} 3$. The variation between $\mathrm{BCE}$ in D3 and D1 $(\triangle B C E)$ was analyzed as a continuous variable, in an exploratory analysis. A receiver-operating characteristic (ROC) curve was performed to evaluate the accuracy of $\triangle \mathrm{BCE}$ to predict 6-month mortality. The optimal cut-off point was mathematically defined using the Youden index [27-31].

Statistical tests were two-tailed with significance defined as a $P$ value less than 0.05 . All $P$ values were two-tailed. We used SPSS version 21.0 (SPSS, Chicago, IL, USA) and R 4.1.0 (R Foundation for Statistical Computing) for all analyses.

\section{Results}

\section{Clinical and epidemiological characteristics}

A total of 90 patients were included in D1, but 15 deceased before D3. A total of 75 patients were included in the D1 and D3 analysis (Fig. 1). The mean age of the patients was $64.8( \pm 15.9)$ years, 42 (56\% were male), and 54 (61\%) with clinical ICU admission. The most frequent foci of sepsis were the lung $(n=41 ; 46 \%)$ and abdominal $(n=36 ; 40 \%)$ infections. The mean SAPS 3 score was $75.8( \pm 12.9)$ points and the mean SOFA score at sepsis diagnosis was $8.5( \pm 3.2)$ points (Table 1$)$. At study period, $32 \%$ of the patients were submitted to hemodialysis and $85 \%$ to mechanical ventilation. Median arterial lactate at septic shock diagnosis was $2.0 \mathrm{mmol} / \mathrm{Dl}$ (IQR 1.2-3.0), and the median volume of fluid resuscitation in septic shock was $44 \mathrm{ml} / \mathrm{kg}$ (IQR 30-65). Fluid balance in the first day of ICU admission was $2874 \mathrm{ml}$ (IQR 1635-5493). Thirty-seven patients (49\%) received hydrocortisone in the septic shock management. Maximum norepinephrine dose in the first day was $0.24 \mu \mathrm{g} / \mathrm{kg} / \mathrm{min}$ (IQR $0.07-0.6124 \mu \mathrm{g} / \mathrm{kg} / \mathrm{min}$ ). 
Table 1 Patient demographic and clinical variables at admission

\begin{tabular}{|c|c|}
\hline Overall population & Distribution and values \\
\hline Patients, $N$ & 90 \\
\hline \multicolumn{2}{|l|}{ Sex } \\
\hline Male & $50(55 \%)$ \\
\hline Female & $40(45 \%)$ \\
\hline Age (years) & $64.8(15.9)$ \\
\hline \multicolumn{2}{|l|}{ Clinical variables } \\
\hline Surgical patients & $35(38 \%)$ \\
\hline \multicolumn{2}{|l|}{ Sepsis foci } \\
\hline Abdomen & $36(40 \%)$ \\
\hline Cutaneous & $2(2 \%)$ \\
\hline Blood & $7(8 \%)$ \\
\hline Urinary & $4(4 \%)$ \\
\hline Lung & $41(46 \%)$ \\
\hline Capillary refill time (s) & $4(2-6)$ \\
\hline Arterial lactate (mmol/L) & $2(1.2-3)$ \\
\hline $\mathrm{SvO} 2(\%)$ & $70(10.2)$ \\
\hline $\mathrm{PaCO}_{2}-\mathrm{PvCO}_{2}(\mathrm{mmHg})$ & $6.5(3.9-10.9)$ \\
\hline SOFA & $8(3.1)$ \\
\hline SAPS 3 & $75.8(12.8)$ \\
\hline \multicolumn{2}{|l|}{ Clinical comorbidities } \\
\hline Solid cancer & $7(8 \%)$ \\
\hline Blood cancer & $7(8 \%)$ \\
\hline HIV & $2(2 \%)$ \\
\hline Cirrhosis & $7(8 \%)$ \\
\hline Chronic kidney disease & $10(11 \%)$ \\
\hline Diabetes & $25(27 \%)$ \\
\hline Hypertension & $32(35 \%)$ \\
\hline
\end{tabular}

Data are $N(\%)$, mean (SD), or median (IQR). SOFA sequential organ failure assessment, SAPS 3 simplified acute physiology score 3

Impact of bioenergetic and clinical variables on mortality

Overall, mitochondrial metabolic endpoints were associated with short- and long-term mortality. The variations (D3-D1) in the mitochondrial respiration sustained by the presence of endogenous intracellular substrates ( $\triangle$ Basal), and the stimulated respiration linked to complex II $(\Delta \mathrm{CII})$ were significantly decreased in non-surviving patients in all timepoints evaluated (Fig. 2A and C, respectively). Further, stimulated respiration linked to complex $\mathrm{I}(\Delta \mathrm{CI})$ was decreased in 6 months non-surviving patients relative to surviving (Fig. 2B). Individual values for mitochondrial oxygen consumption at D1 and D3 at basal, linked to $\mathrm{CI}$ and $\mathrm{CII}$ as well as $\triangle \mathrm{CII}$ were associated with ICU, hospital and 6-month mortality after sepsis diagnosis (Additional file 3: Table S1). Patients that deceased in the ICU presented elevated arterial lactate, C-reactive protein, capillary refill time and SOFA scores at D1. These patients also had increased SOFA score at and D3 compared with those that survived (Table 2). BCE at D1 and D3 were reduced in non-surviving patients. The number of patients that improved SOFA $(\triangle \mathrm{SOFA}<0 ; 11$ [34\%] versus $38[88 \%], P<0.001)$ and $\mathrm{BCE}(\triangle \mathrm{BCE}>0$; $2[6.3 \%]$ versus $33[77 \%], P<0.001)$ was lower in nonsurvivors (Table 2). Patients that deceased during hospital admission had increased arterial lactate, SOFA at D1 and D3, capillary refill time and SAPS 3. BCE 

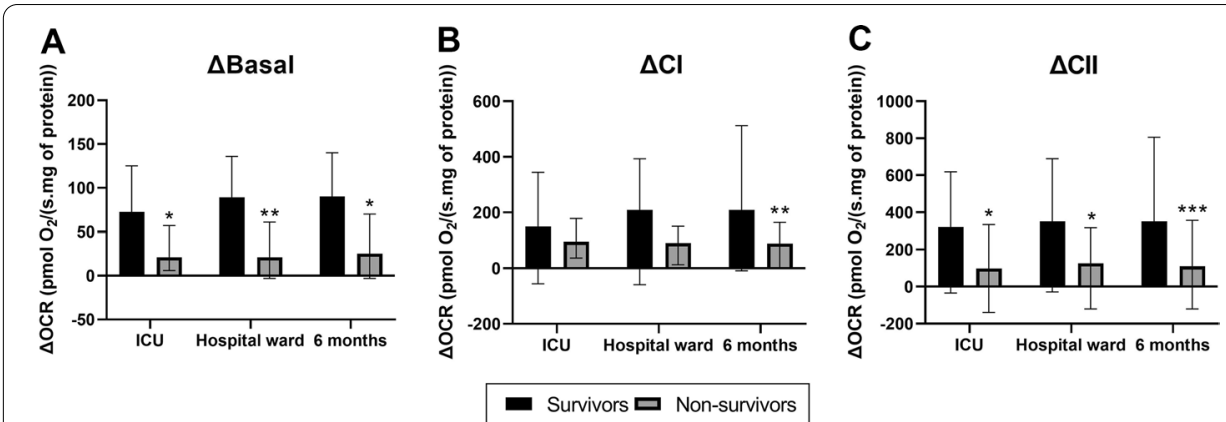

Fig. 2 Mitochondrial bioenergetic function is reduced in lymphocytes of non-surviving septic shock patients. Variations (day 3 minus day 1 admission) in mitochondrial oxygen consumption rates in conditions not stimulated by metabolic substrates $(\mathbf{A}, \triangle B$ Basal), stimulated by complex I substrates, pyruvate, malate, and glutamate $(\mathbf{B}, \Delta \mathrm{Cl})$, and stimulated by complex II substrate, succinate $(\mathbf{C}, \Delta \mathrm{CII})$. Figures are presented as median $\pm I Q R$. ${ }^{*}$ Indicates significant differences relative to surviving at $P<0.05 ;{ }^{* *} P<0.01$; and ${ }^{* * *} P<0.001$

at D1 and D3 are lower in non-surviving than surviving patients. Survivors had greater incidence of improvement in SOFA $(\triangle \mathrm{SOFA}<0)$, as well as in $\mathrm{BCE}(\triangle \mathrm{BCE}>0)$ than nonsurvivors (Table 2).

Patients who died within 6 months after septic shock presented elevated arterial lactate levels at ICU admission and increased SAPS 3, and had the highest SOFA scores at D1 and D3. Survivors also had greater incidence of improvement in SOFA $(\triangle \mathrm{SOFA}<0)$ and in $\mathrm{BCE}(\triangle \mathrm{BCE}>0)$ than nonsurvivors (Table 2). Remarkably, after adjusting for possible confounders, the 6-month mortality rate was lower in patients who had an improved BCE at D3 (RR 0.38, CI 95\% 0.18-0.78; $P<0.001$ ), while classic laboratory and clinical variables such as lactate, CRP, SOFA and SAPS 3 were not associated with survival or death (Table 3 ).

$\mathrm{BCE}$ at D1 was inversely associated with SOFA (Spearman $=-0.28 ; P=0.005)$, but had no associations with arterial lactate (Spearman $=-0.2 ; P=0.057$ ) or with plasma $\mathrm{C}$-reactive protein levels $(\mathrm{CRP})($ Spearman $=0.007 ; P=0.947)$. Six patients $(8 \%)$ developed a need for a tracheostomy during ICU admission. These patients worsened in the $\triangle \mathrm{BCE}$ when compared to those who did not need a tracheostomy $(-0.085 \pm 0.1$ versus $0.048 \pm 0.14$, respectively; $P=0.11$ ), but without reaching statistical significance. Thirtysix patients developed a new infection after the initial injury, and these patients had no statistically significant difference in their median $\triangle B C E$ when compared to those who did not developed reinfection $(-0.008 \pm 0.23$ versus $0.089 \pm 0.19$, respectively, $P=0.29$ ). Patients who needed MV had lower values of $\triangle \mathrm{BCE}$ when compared with those who did not need it, reaching statistical significance: $0.006 \pm 0.14$ versus $0.1 \pm 0.13$, respectively; $P=0.05$. Patients who needed hemodialysis had worsening in the $\triangle \mathrm{BCE}$, while those who did not need hemodialysis had an improvement in their values $(-0.029 \pm 0.1$ versus $0.07 \pm 0.2 ; P<0.01)$. Patients who improved $\triangle \mathrm{BCE}(\triangle \mathrm{BCE}>0)$ had a shorter ICU length of stay when compared with those who impaired $\triangle \mathrm{BCE}(\triangle \mathrm{BCE}<0): 6 \pm 4$ days versus $9.5 \pm 14$ days, respectively $(P=0.02)$. The AUROC for $\triangle B C E$ as prognostic biomarker of 6-month mortality was 0.90 (95\% CI 0.83-0.91, $P<0.01)$ (see Additional file 2: Figure S2). A cut-off point of -0.002 in $\triangle \mathrm{BCE}$ had a $100 \%$ of sensitivity and $73 \%$ of specificity for the outcome (Youden's J Index $=0.73$ ). Distinct cut-off values of $\triangle \mathrm{BCE}$ relative to sensitivity and specificity, and Youden's J Index are shown in Table 4. 
Table 2 Univariate analysis of variables associated with ICU, hospital and 6-month mortality

\begin{tabular}{|c|c|c|c|c|c|c|c|c|c|}
\hline \multirow[t]{2}{*}{ Variables } & \multicolumn{2}{|l|}{ ICU } & \multirow[t]{2}{*}{ P } & \multicolumn{2}{|l|}{ Hospital } & \multirow[t]{2}{*}{ P } & \multicolumn{2}{|l|}{ Six months } & \multirow[t]{2}{*}{ P } \\
\hline & $\begin{array}{l}\text { Survivors } \\
n=43\end{array}$ & $\begin{array}{l}\text { Non- } \\
\text { survivors } \\
n=32\end{array}$ & & $\begin{array}{l}\text { Survivors } \\
n=32\end{array}$ & $\begin{array}{l}\text { Non- } \\
\text { survivors } \\
n=43\end{array}$ & & $\begin{array}{l}\text { Survivors } \\
n=25\end{array}$ & $\begin{array}{l}\text { Non- } \\
\text { survivors } \\
n=50\end{array}$ & \\
\hline Age & $64.14(18.1)$ & $67.16(14.7)$ & 0.443 & $61.97(16.5)$ & $68.00(16.6)$ & 0.122 & $62.1(14.0)$ & $67.1(17.8)$ & 0.228 \\
\hline $\begin{array}{l}\text { Clinical } \\
\text { admis- } \\
\text { sion at } \\
\text { ICU }\end{array}$ & 25 (58\%) & $21(66 \%)$ & 0.51 & $21(66 \%)$ & 25 (58\%) & 0.51 & 16 (64\%) & $30(60 \%)$ & 0.737 \\
\hline Sex (male) & $26(60 \%)$ & $16(50 \%)$ & 0.366 & $18(57 \%)$ & $24(56 \%)$ & 0.97 & 15 (60\%) & $27(54 \%)$ & 0.622 \\
\hline $\begin{array}{l}\text { Pulmonary } \\
\text { sepsis }\end{array}$ & $21(51 \%)$ & 12 (38\%) & 0.24 & $18(56 \%)$ & $16(37 \%)$ & 0.101 & $13(52 \%)$ & $21(42 \%)$ & 0.412 \\
\hline $\begin{array}{l}\text { Solid neo- } \\
\text { plasia }\end{array}$ & $13(30 \%)$ & $4(11 \%)$ & 0.07 & $9(28 \%)$ & $8(18 \%)$ & 0.33 & $6(24 \%)$ & $11(22 \%)$ & 0.845 \\
\hline $\begin{array}{l}\text { Hemato- } \\
\text { logical } \\
\text { neoplasia }\end{array}$ & $2(4.6 \%)$ & $3(9.4 \%)$ & 0.417 & 1 (3.1\%) & $4(9.3 \%)$ & 0.386 & $0(0 \%)$ & $5(10 \%)$ & 0.162 \\
\hline Cirrhosis & $1(2.3 \%)$ & $4(13 \%)$ & 0.081 & $1(3.1 \%)$ & $4(9.3 \%)$ & 0.386 & $1(4.0 \%)$ & $4(8.0 \%)$ & 0.66 \\
\hline COPD & $7(16 \%)$ & $4(13 \%)$ & 0.647 & $5(16 \%)$ & $6(14 \%)$ & 0.84 & $3(12.0 \%)$ & $8(16 \%)$ & 0.742 \\
\hline CKD & $5(12 \%)$ & $2(6.2 \%)$ & 0.428 & $5(16 \%)$ & $2(4.6 \%)$ & 0.11 & $4(16 \%)$ & $3(6 \%)$ & 0.161 \\
\hline $\begin{array}{c}\text { Chronic } \\
\text { hyper- } \\
\text { tension }\end{array}$ & $11(26 \%)$ & $13(41 \%)$ & 0.167 & $9(28 \%)$ & 15 (35\%) & 0.534 & $7(28 \%)$ & 17 (34\%) & 0.6 \\
\hline Diabetes & $11(26 \%)$ & $10(31 \%)$ & 0.589 & $10(32 \%)$ & $11(26 \%)$ & 0.589 & $9(36 \%)$ & $12(24 \%)$ & 0.275 \\
\hline $\mathrm{SvO}_{2}(\%)$ & $68.01(6.1)$ & $71.36(11.6)$ & 0.215 & $67.71(9.0)$ & $70.70(11.1)$ & 0.266 & $68.18(9.1)$ & 70.06 (10.9) & 0.504 \\
\hline $\begin{array}{l}\text { Lactate D1 } \\
\qquad(\mathrm{mmol} / \mathrm{L})\end{array}$ & $1.4(1.1-2.1)$ & $2.8(2.0-4.0)$ & 0.002 & $1.4(1.1-1.9$ & $2.55(1.7-3.6$ & 0.015 & $1.4(0.9-1.6)$ & $2.2(1.6-3.5)$ & 0.041 \\
\hline SOFA D1 & $7.63(3.0)$ & $1.0(2.4)$ & $<0.0001$ & $7.25(2.55)$ & $9.67(2.95)$ & $<0.0001$ & $7.40(2.6)$ & $9.26(3.0)$ & 0.011 \\
\hline SOFA D3 & $3(2-5)$ & $12.5(7-19.5)$ & $<0.0001$ & $3(1.7-5)$ & $9(4-16.5)$ & $<0.0001$ & $3(1-4)$ & $\begin{array}{c}7.5 \\
(3.25-15.5)\end{array}$ & $<0.0001$ \\
\hline$\triangle S O F A<0$ & $38(88 \%)$ & $11(34 \%)$ & $<0.0001$ & $28(88 \%)$ & $21(49 \%)$ & $<0.0001$ & $22(88 \%)$ & $27(54 \%)$ & 0.004 \\
\hline SAPS 3 & $73.98(13.2)$ & $78.60(12.1)$ & 0.1 & $72.16(13.1)$ & $78.77(12.0)$ & 0.026 & $70.68(11.2)$ & $78.58(12.9)$ & 0.011 \\
\hline $\mathrm{CRP}(\mathrm{mg} / \mathrm{L})$ & $\begin{array}{c}153 \\
(69.7-216)\end{array}$ & $\begin{array}{c}220 \\
(119-284)\end{array}$ & 0.02 & $153(69-223)$ & $\begin{array}{c}196 \\
(119-269)\end{array}$ & 0.062 & $\begin{array}{l}153(72.6- \\
209)\end{array}$ & $188(98-258)$ & 0.076 \\
\hline CRT D1 (s) & $4(2-4)$ & $5.5(3-8)$ & 0.04 & $3.5(2-4)$ & $4(3-7)$ & 0.046 & $4(3-4)$ & $4(2.25-7)$ & 0.319 \\
\hline BCE D1 & $0.354(0.1)$ & $0.243(0.1)$ & $<0.0001$ & $0.378(0.1)$ & $0.254(0.1)$ & $<0.0001$ & $0.380(0.1)$ & $0.270(0.1)$ & $<0.0001$ \\
\hline BCE D3 & $0.456(0.18)$ & $0.210(0.1)$ & $<0.0001$ & $0.535(0.1)$ & $0.215(0.1)$ & $<0.0001$ & $0.543(0.1)$ & $0.267(0.2)$ & $<0.0001$ \\
\hline$\triangle B C E>0$ & $33(77 \%)$ & $2(6.3 \%)$ & $<0.0001$ & 31 (97\%) & $4(9.3 \%)$ & $<0.0001$ & 24 (96\%) & 11 (22\%) & $<0.0001$ \\
\hline
\end{tabular}

Table 3 Multivariate analysis of variables associated with 6-month mortality

\begin{tabular}{lcc}
\hline Variables & Multivariate analysis & $P$ \\
\hline Hematological neoplasia & RR $1.1(0.37-3.26)$ & 0.86 \\
CKD & RR $0.75(0.22-2.48)$ & 0.64 \\
Lactate-D1 & RR $1.01(0.88-1.14)$ & 0.88 \\
$\triangle S O F A<0$ & RR $1.01(0.49-2.08)$ & 0.96 \\
SAPS 3 & RR $1.01(0.98-1.03)$ & 0.321 \\
$\triangle B C E>0$ & RR $0.38(0.18-0.78)$ & 0.009 \\
\hline
\end{tabular}

$\triangle$ mathematically indicates the pairwise variation between values obtained at day 3 (D3) minus day 1 (D1), for the indicated variable. SOFA sequential organ failure assessment, SAPS 3 simplified acute physiology score 3, BCE biochemical coupling efficiency, $C K D$ chronic kidney disease, $R R$ risk ratio 
Table 4 Sensitivity, specificity and Youden's J index for measurements of $\triangle B C E$

\begin{tabular}{llll}
\hline$\Delta$ BCE & Sensitivity & Specificity & $\begin{array}{l}\text { Youden's } \\
\text { J Index }\end{array}$ \\
\hline-0.220 & $100 \%$ & $0 \%$ & 0 \\
-0.111 & $98 \%$ & $31 \%$ & 0.31 \\
-0.002 & $100 \%$ & $73 \%$ & 0.73 \\
0.11 & $70 \%$ & $88 \%$ & 0.58 \\
0.219 & $33 \%$ & $97 \%$ & 0.31 \\
0.354 & $3 \%$ & $100 \%$ & 0.03 \\
\hline
\end{tabular}

\section{Discussion}

Our findings highlight a high short- and long-term mortality among septic shock patients. Mitochondrial metabolism in the lymphocytes of these patients provided a particular signature for the deterioration of clinical outcomes. Among the clinical and mitochondrial metabolic endpoints, $\triangle \mathrm{BCE}$ was independently associated with longterm mortality in our cohort.

Although SOFA is a well-validated instrument to estimate mortality risk, the addition of new and more sensitive laboratory variables to this score may benefit its predictive validity. Indeed, in the "The Third International Consensus Definitions for Sepsis and Septic Shock (Sepsis-3)" [1], Kramer suggests the incorporation of new candidate biochemical markers to improve the SOFA score sensitivity. Such an approach is pertinent because it may provide earlier information to support clinical decisions, and thus, patients are more likely to achieve better clinical outcomes that reflect their prognosis beyond the ICU $[1,32]$. In this context, our approach does not rule out the clinical relevance of SOFA or other clinical scores, but proposes "a look" to a promising complementary prognostic tool. Physiologically, the mitochondria are capable of adjusting their metabolic energy demands and signals to protect cells from insults [7, 9, 14]. This has been clinically investigated in immune cells of patients with sepsis and in septic shock, to estimate the influence of bioenergetics on inflammatory responsiveness, severity of symptoms and the deterioration of health status due to organ failure [9, 33, 34]. Such relevance is conceptually well-illustrated by proposals that activated immune cells can undergo complex plasticity phenomena, which is sustained by mitochondria, "the powerhouses of immunity" [6]. Accordingly, it was demonstrated in blood mononuclear cells that mitochondrial dysfunction and damage progress over time, along with the severity of symptoms, albeit strong conclusions were limited by the small number of patients $[6$, 9, 34].

Here, we showed an improved metabolism from D1 to D3 $(\triangle \mathrm{BCE})$ admission to the ICU which paralleled survival, implying that increments in mitochondrial bioenergetic function may reflect metabolic reprograming leading to improvements of the general health status in septic shock patients. Another study found that an early normalization of mitochondrial biogenesis genes expression profile accelerated sepsis recovery and shortened the time in the ICU [34], thus reinforcing the suggestion of clinical and functional relevance in approaching mitochondrial phenotypes. Although we do not address gene-profile, our study conciliates the mechanistic concept that an increased biogenesis governs plasticity mechanisms directed to renew damaged mitochondria, and as 
consequence, increase energetic efficiency. On the other hand, some intrinsic features support the investigation of mitochondrial bioenergetic effectors in immune cells as biomarkers of outcomes. For instance, dysregulation of immune cells, including lymphocytes, are key components of inflammatory overreaction and organ failure, considered as hallmarks of septic shock; and as resident cells in the circulation, they survey whole body microenvironments, are cells easy to sample and to perform metabolic profiling in routine intensive care practice. The association between improved mitochondrial function with clinically relevant outcomes, such as length of stay in the ICU, the need for mechanical ventilation and the need for hemodialysis, reinforces this hypothesis.

The other clinically relevant spectrum of the sepsis, the compensatory anti-inflammatory response syndrome (CARS), is an event that occurs in a subgroup of septic patients that develop profound acquired immunosuppression. Such condition may cause difficulties to efficiently eradicate the primary infection despite patients are submitted to standardized treatment protocols [35]. Hence, it is important to take into consideration that sepsis leads to a complex immune response that evolves over time, with the simultaneous implication of both, proinflammatory and anti-inflammatory mechanisms for the clinical outcomes [36]. The intensity and duration of the mentioned exacerbated anti-inflammatory phase seem to be closely related with the development of nosocomial infections. Also, an immunometabolic reprogramming in response to sepsis may rely on the interplay between the energy demands and capacity of lymphocyte mitochondria to produce ATP [37]. However, whether or not a metabolic reprograming, here mirrored by $\mathrm{BCE}$, exerts a significant influence in the state of immunosuppression remains to be explored [38]. It is known that the lymphocytes challenged by sepsis displayed an exhausted-like phenotype characterized by decreased functions including the capacity of proliferation, cytokine production and increased coinhibitory receptor expression, culminating in apoptotic cell death [35]. Considering that it is not completely known the exact extrinsic and intrinsic factors that determine the severity of lymphocytes exhaustion, and likely immunosuppression, we tend to suggest decreased mitochondrial bioenergetics as an active player. Accordingly, in physiological conditions lymphocytes are highly oxidative cells which implies in immunological processes highly dependent of an appropriate ATP support. Studies have shown a bidirectional interaction between mitochondria and molecular inflammatory effectors that drive cell responses to an infection. This crosstalk between immunogenic effectors and mitochondrial activity involves tolllike receptors, damage-associated molecular patterns (DAMP's), pathogen-associated molecular patterns (PAMP's); and NLRP3 inflammasome activation, all driving mitochondrial mechanisms such as increased reactive oxygen species production, and lymphocyte cytokines release $[39,40]$.

To expand the exploratory analysis of our study, we found that patients who did not develop a secondary infection, who theoretically would be less susceptible to immunosuppression, had greater increases in BCE when compared to those who had a secondary infection. However, this result was not statistically significant and the sample size was not designed for this analysis, being any conclusions based on these observations merely conjectures that deserve attention in future studies.

Moreover, in cases of unresolved inflammatory-related immunosuppression activity, this condition may progress to a persistent inflammation-immunosuppression 
and catabolism syndrome (PICS), that often evolve to a chronic critical illness state [41]. A hallmark of this condition is the need for a tracheostomy during the course of septic shock, that is associated with impairment in adaptive immunity related to lymphocytes [42]. In our study, patients who needed a tracheostomy had a worsening in BCE (data no shown), when compared with those who did not. Despite this result did not reach statistical significance, we believe that the small number of tracheostomized patients in our sample could explain this fact.

Our study has the merits of enrolling a large number of septic shock patients in which the respiratory abnormalities in specific mitochondrial complexes represent immunometabolic targets associated with disease pathogenesis. Indeed, no previous study has integrated the activity of mitochondrial complexes $\mathrm{CI}, \mathrm{CII}$, and $\mathrm{CV}$, and $\mathrm{BCE}$ in lymphocytes using two time-points, which allowed us to estimate metabolic reprogramming relative to the disease progression. The resulting improvement or worsening of the mitochondrial respiratory endpoints in the ICU highlight potential immunometabolic mechanistic targets, and also provide perspective regarding how metabolic reprogramming influences clinical outcomes. This approach outlined BCE as a candidate prognostic biomarker to be incorporated into clinical practice to support therapeutic management and decision-making. Although we provided promising insights regarding mitochondria bioenergetics as biomarker, this approach still requires advances to reach a clinical applicability. The development of ready-to-use assays may increase the potentialities of clinical utilization, nonetheless before pursuing this goal, it is imperative to identify specific mitochondrial functions implicated in the response to the disease.

Our study has a number of limitations that should be further explored. It is a unicentric study, which may limit the external validity of the main findings. Second, it is a population that presented with a high risk of mortality independent of the septic shock. Even so, the mortality reported here reflects the severity measured by the SAPS 3 score, and it is similar to previous studies performed in Brazil and worldwide [43-45]. Third, we evaluated lymphocytes as a whole, albeit different types of lymphocytes may have specific metabolic phenotypes; however, such specificity did not interfere with the predictive nature of $\triangle \mathrm{BCE}$. Indeed, the sensitivity and specificity $\triangle B C E$ assessed with a ROC curve further confirmed its potential prognostic value for 6- month mortality. As a future perspective, it is important to investigate associations with other clinically relevant outcomes, such as clinical frailty, quality of life, long-term functional status, secondary immunosuppression, incidence of PICS and chronic organ failure after septic shock.

\section{Conclusions}

Our work highlights that impaired mitochondrial capacity to improve BCE provided an earlier recognition of septic shock patients in the ICU at risk of deterioration of health status. On the contrary, improved BCE capacity was associated with favorable clinical outcomes. Further research is needed to outline BCE as a prognostic biomarker to be incorporated into intensive care setting. 


\section{Abbreviations}

ICU: Intensive care unit; SOFA: Sequential organ failure assessment; SAPS 3: Simplified acute physiology score 3; D1: Day first admission ICU; D3: Day 3 admission ICU; $\triangle$ : Values obtained at day 3 minus day 1; Cl: Mitochondrial complex I; Cll: Mitochondrial complex II; BCE: Biochemical coupling efficiency; IQR: Interquartile range.

\section{Supplementary Information}

The online version contains supplementary material available at https://doi.org/10.1186/s40635-021-00404-9.

Additional file 1: Figure S1. Respirometry curve profile. Representative image of a respirometry assay performed in lymphocytes from a control subject.

Additional file 2: Figure S2. Receiver-operating characteristic curve. The BCE at a cut-off value of -0.002 (indicated by the black dot) represents 100\% sensitivity and $73 \%$ specificity for predicting 6 -month mortality. The obtained area under the curve was 0.90 .

Additional file 3: Table S1. Mitochondrial respiratory rates in lymphocytes of survival and nonsurvival patients.

\section{Acknowledgements}

The authors thank Dr. Guilherme Mazzini for his scientific support.

\section{Authors' contributions}

Dr. Portela, Dr. Nedel and Dr. Galina had full access to all the data in the study and take responsibility for the integrity of the data and the accuracy of the data analysis. WLN, TLH, LVP, and AG built the study concept. THMM, and JAJ were involved in the patients' clinical management, and collected the samples and data. LVP, M De B, and WLN designed the study and investigation. MSR, AK, and NRS prepared samples, performed the mitochondrial assays, analyzed the data and created the figures. WLN and LVP wrote the first draft of the manuscript, and all authors reviewed and edited the final manuscript. LVP acquired funding. All authors read and approved the final manuscript.

\section{Funding}

This study was supported by resources from Brazilian Scientific Agencies FAPERGS \#1010267; FAPERGS/PPSUS\#17/25510001; FAPERGS/PRONEX\#16/2551-0000499-4, CAPES/PNPD\#1663; and CNPq INNT \#5465346/2014-6. The funding body did not take any role in the design of the study and collection, analysis, and interpretation of data and in writing the manuscript.

\section{Availability of data and materials}

Individual participant data that underlie the results reported in this article, after deidentification, will be made available to researchers who provide a methodologically sound proposal for analyses to achieve aims in the approved proposal, immediately following article publication. Please address the proposals directly to roskaportela@gmail.com.

\section{Declarations}

\section{Ethics approval and consent to participate}

Ethics approval was obtained from the institutional ethics committees from the Hospital Conçeição, Porto Alegre, RS, Brazil. The study was registered at Plataforma Brazil (66240017.0.0000.5530). Consent waiver was granted by the ethics committee for the collection and use of participants' personal information.

\section{Consent for publication}

Not applicable.

\section{Competing interests}

The authors declare that they have no competing interests.

\section{Author details}

'Laboratory of Neurotrauma and Biomarkers, Departamento de Bioquímica, Programa de Pós-Graduação em Bioquímica, ICBS, Universidade Federal do Rio Grande do Sul, Rua Ramiro Barcelos, 2600, anexo, Porto Alegre, RS, Brazil. ${ }^{2}$ Intensive Care Unit, Hospital Nossa Senhora da Conceição, Grupo Hospitalar Conceição, Porto Alegre, RS, Brazil. 'Zimmer Lab, Departamento de Bioquímica, Universidade Federal do Rio Grande do Sul, Porto Alegre, RS, Brazil. ${ }^{4}$ Laboratory of Bioenergetics and Mitochondrial Physiology, Instituto de Bioquímica Médica Leopoldo de Meis, Universidade Federal do Rio de Janeiro (UFRJ), Rio de Janeiro, RJ, Brazil. ${ }^{5}$ Program in Integrative Cell Signaling and Neurobiology of Metabolism, Department of Comparative Medicine, Yale University School of Medicine, New Haven, CT, USA.

Received: 2 March 2021 Accepted: 10 July 2021

Published online: 24 July 2021 


\section{References}

1. Singer M, Deutschman CS, Seymour CW et al (2016) The third international consensus definitions for sepsis and septic shock (sepsis-3). JAMA 315:801. https://doi.org/10.1001/jama.2016.0287

2. Dellinger RP, Levy MM, Rhodes A et al (2013) Surviving sepsis campaign: International guidelines for management of severe sepsis and septic shock, 2012. Intensive Care Med 39:165-228. https://doi.org/10.1007/s00134-012-2769-8

3. Singer M (2017) Critical illness and flat batteries. Crit Care 21:309. https://doi.org/10.1186/s13054-017-1913-9

4. Carré JE, Orban J-C, Re L et al (2010) Survival in critical illness is associated with early activation of mitochondrial biogenesis. Am J Respir Crit Care Med 182:745-751. https://doi.org/10.1164/rccm.201003-03260C

5. Brealey D, Brand M, Hargreaves I et al (2002) Association between mitochondrial dysfunction and severity and outcome of septic shock. Lancet 360:219-223. https://doi.org/10.1016/S0140-6736(02)09459-X

6. Mills EL, Kelly B, O'Neill LAJ (2017) Mitochondria are the powerhouses of immunity. Nat Immunol 18:488-498. https://doi.org/10.1038/ni.3704

7. Cheng S-C, Scicluna BP, Arts RJW et al (2016) Broad defects in the energy metabolism of leukocytes underlie immunoparalysis in sepsis. Nat Immunol 17:406-413. https://doi.org/10.1038/ni.3398

8. Pearce EJ, Pearce EL (2018) Driving immunity: all roads lead to metabolism. Nat Rev Immunol 18:81-82. https://doi. org/10.1038/nri.2017.139

9. Kramer PA, Ravi S, Chacko B et al (2014) A review of the mitochondrial and glycolytic metabolism in human platelets and leukocytes: Implications for their use as bioenergetic biomarkers. Redox Biol 2:206-210

10. Gotts JE, Matthay MA (2016) Sepsis: pathophysiology and clinical management. BMJ 353:11585. https://doi.org/10 1136/bmj.i1585

11. Puskarich MA, Kline JA, Watts JA et al (2016) Early alterations in platelet mitochondrial function are associated with survival and organ failure in patients with septic shock. J Crit Care 31:63-67. https://doi.org/10.1016/j.jcrc.2015.10. 005

12. Sjovall F, Morota S, Hansson MJ et al (2010) Temporal increase of platelet mitochondrial respiration is negatively associated with clinical outcome in patients with sepsis. Crit Care 14:R214. https://doi.org/10.1186/cc9337

13. Sjövall F, Morota S, Persson J et al (2013) Patients with sepsis exhibit increased mitochondrial respiratory capacity in peripheral blood immune cells. Crit Care 17:R152. https://doi.org/10.1186/cc12831

14. Jang DH, Greenwood JC, Spyres MB, Eckmann DM (2017) Measurement of mitochondrial respiration and motility in acute care. J Intensive Care Med 32:86-94. https://doi.org/10.1177/0885066616658449

15. Makrecka-Kuka M, Krumschnabel G, Gnaiger E (2015) High-resolution respirometry for simultaneous measurement of oxygen and hydrogen peroxide fluxes in permeabilized cells, tissue homogenate and isolated mitochondria. Biomolecules 5:1319-1338. https://doi.org/10.3390/biom5031319

16. Gnaiger E (2014) Mitochondrial Pathways and Respiratory Control An Introduction to OXPHOS Analysis, 4th Ed. OROBOROS MiPNet Publications, Innsbruck

17. Japiassú AM, Santiago APSA, D'Avila JDCP et al (2011) Bioenergetic failure of human peripheral blood monocytes in patients with septic shock is mediated by reduced F1Fo adenosine-5'-triphosphate synthase activity. Crit Care Med 39:1056-1063. https://doi.org/10.1097/CCM.0b013e31820eda5c

18. Levy MM, Fink MP, Marshall JC et al (2003) 2001 SCCM/ESICM/ACCP/ATS/SIS international sepsis definitions conference. Intensive Care Med 29:530-538. https://doi.org/10.1007/s00134-003-1662-x

19. Vincent JL, Moreno R, Takala J et al (1996) The SOFA (Sepsis-related Organ Failure Assessment) score to describe organ dysfunction/failure. On behalf of the Working Group on sepsis-related problems of the European society of intensive care medicine. Intensive Care Med 22:707-710

20. Moreno RP, Metnitz PGH, Almeida E et al (2005) SAPS 3-from evaluation of the patient to evaluation of the intensive care unit. Part 2: development of a prognostic model for hospital mortality at ICU admission. Intensive Care Med 31:1345-1355. https://doi.org/10.1007/s00134-005-2763-5

21. Lambden S, Laterre PF, Levy MM, Francois B (2019) The SOFA score-development, utility and challenges of accurate assessment in clinical trials. Crit Care 23:374. https://doi.org/10.1186/s13054-019-2663-7

22. de Grooth H-J, Geenen IL, Girbes AR et al (2017) SOFA and mortality endpoints in randomized controlled trials: a systematic review and meta-regression analysis. Crit Care 21:38. https://doi.org/10.1186/s13054-017-1609-1

23. Karakike E, Kyriazopoulou E, Tsangaris I et al (2019) The early change of SOFA score as a prognostic marker of 28-day sepsis mortality: analysis through a derivation and a validation cohort. Crit Care 23:387. https://doi.org/10.1186/ s13054-019-2665-5

24. Dellinger RP, Levy MM, Rhodes A et al (2013) Surviving sepsis campaign: international guidelines for management of severe sepsis and septic shock: 2012. Crit Care Med 41:580-637. https://doi.org/10.1097/CCM.0b013e31827e83af

25. Pecina P, Houšt'ková H, Mráček T et al (2014) Noninvasive diagnostics of mitochondrial disorders in isolated lymphocytes with high resolution respirometry. BBA Clin 2:62-71. https://doi.org/10.1016/j.bbacli.2014.09.003

26. Carteri RB, Kopczynski A, Rodolphi MS et al (2019) Testosterone administration after traumatic brain injury reduces mitochondrial dysfunction and neurodegeneration. J Neurotrauma 36:2246-2259. https://doi.org/10.1089/neu. 2018.6266

27. López-Ratón M, Rodríguez-Álvarez MX, Suárez CC, Sampedro FG (2014) OptimalCutpoints: an R package for selecting optimal cutpoints in diagnostic tests. J Stat Softw 61:1-36. https://doi.org/10.18637/jss.v061.i08

28. Aoki K, Misumi J, Kimura T et al (1997) Evaluation of cutoff levels for screening of gastric cancer using serum pepsinogens and distributions of levels of serum pepsinogen I, II and of PG I / PG II ratios in a gastric cancer casecontrol study. J Epidemiol 7:143-151. https://doi.org/10.2188/jea.7.143

29. Greiner M, Pfeiffer D, Smith R (2000) Principles and practical application of the receiver-operating characteristic analysis for diagnostic tests. Prev Vet Med 45:23-41. https://doi.org/10.1016/S0167-5877(00)00115-X

30. Youden WJ (1950) Index for rating diagnostic tests. Cancer 3:32-35. https://doi.org/10.1002/1097-0142(1950)3:1\% 3c32::AID-CNCR2820030106\%3e3.0.CO;2-3

31. Shapiro DE (1999) The interpretation of diagnostic tests. Stat Methods Med Res 8:113-134. https://doi.org/10.1191/ 096228099666928387 
32. Shankar-Hari M, Phillips GS, Levy ML et al (2016) Developing a new definition and assessing new clinical criteria for septic shock. JAMA 315:775. https://doi.org/10.1001/jama.2016.0289

33. Ganeshan K, Nikkanen J, Man K et al (2019) Energetic trade-offs and hypometabolic states promote disease tolerance. Cell 177:399-413.e12. https://doi.org/10.1016/j.cell.2019.01.050

34. Kraft BD, Chen L, Suliman HB et al (2019) Peripheral blood mononuclear cells demonstrate mitochondrial damage clearance during sepsis. Crit Care Med 47:651-658. https://doi.org/10.1097/CCM.0000000000003681

35. Venet F, Rimmelé T, Monneret G (2018) Management of sepsis-induced immunosuppression. Crit Care Clin 34:97-106

36. Hotchkiss RS, Monneret G, Payen D (2013) Sepsis-induced immunosuppression: from cellular dysfunctions to immunotherapy. Nat Rev Immunol 13:862-874

37. Pool R, Gomez H, Kellum JA (2018) Mechanisms of organ dysfunction in sepsis. Crit Care Clin 34:63-80. https://doi. org/10.1016/j.ccc.2017.08.003

38. Venet F, Davin F, Guignant C et al (2010) Early assessment of leukocyte alterations at diagnosis of septic shock. Shock 34:358-363. https://doi.org/10.1097/SHK.0b013e3181dc0977

39. Missiroli S, Genovese I, Perrone M et al (2020) The role of mitochondria in inflammation: from cancer to neurodegenerative disorders. J Clin Med 9:740. https://doi.org/10.3390/jcm9030740

40. Cloonan SM, Choi AMK (2013) Mitochondria: sensors and mediators of innate immune receptor signaling. Curr Opin Microbiol 16:327-338. https://doi.org/10.1016/j.mib.2013.05.005

41. Mira JC, Gentile LF, Mathias BJ et al (2017) Sepsis pathophysiology, chronic critical illness, and persistent inflammation-immunosuppression and catabolism syndrome. Crit Care Med 45:253-262. https://doi.org/10.1097/CCM.00000 00000002074

42. Mira JC, Brakenridge SC, Moldawer LL, Moore FA (2017) Persistent inflammation, immunosuppression and catabolism syndrome. Crit Care Clin 33:245-258. https://doi.org/10.1016/j.ccc.2016.12.001

43. Buchman TG, Simpson SQ, Sciarretta KL et al (2020) Sepsis among medicare beneficiaries. Crit Care Med 48:276-288, https://doi.org/10.1097/CCM.0000000000004224

44. Machado FR, Cavalcanti AB, Bozza FA et al (2017) The epidemiology of sepsis in Brazilian intensive care units (the Sepsis PREvalence Assessment Database, SPREAD): an observational study. Lancet Infect Dis 17:1180-1189. https:// doi.org/10.1016/S1473-3099(17)30322-5

45. Conde KAP, Silva E, Silva CO et al (2013) Differences in sepsis treatment and outcomes between public and private hospitals in Brazil: a multicenter observational study. PLOS ONE 8:e64790. https://doi.org/10.1371/journal.pone. 0064790

\section{Publisher's Note}

Springer Nature remains neutral with regard to jurisdictional claims in published maps and institutional affiliations.

\section{Submit your manuscript to a SpringerOpen ${ }^{\circ}$ journal and benefit from:}

- Convenient online submission

Rigorous peer review

- Open access: articles freely available online

- High visibility within the field

Retaining the copyright to your article

Submit your next manuscript at $\gg$ springeropen.com 